\title{
Selective Maintenance for Multistate Series Systems With S-Dependent Components
}

\author{
Cuong Duc Dao, Student Member, IEEE, and Ming J. Zuo, Senior Member, IEEE
}

\begin{abstract}
In this paper, we will consider the selective maintenance problem for multistate series systems with stochastic dependent components. In multistate systems, the health state of a component may vary from perfect functioning to complete failure. The stochastic dependence (S-dependence) between components is discussed and categorized into two types in multistate context. First, the failure of a component can immediately cause complete failures of some other components in the system. Second, as components deteriorate, the reduced working performance rate of a multistate component affects the state as well as the degradation rate of its subsequent components in series structure. The system reliability is evaluated using an approach based on stochastic process. A costbased selective maintenance model is developed for the multistate system with $S$-dependent components to maximize the total system profit, which includes the production gain and loss in the next mission as well as possible maintenance costs for the system. Analyses of systems with independent and dependent components are provided. It is observed that ignoring S-dependence in the system may lead to alternative maintenance decision making and an optimistic estimation of the system performance.
\end{abstract}

Index Terms-Failure interaction, multicomponent systems, multistate component, multistate systems, selective maintenance, stochastic dependence (S-dependence).

\section{ACRONYMS}

MSS Multistate systems.

IF Immediate failure.

IFD Immediate failure dependence.

GDD Gradual degradation dependence.

C-K Chapman-Kolmogorov.

SM Selective maintenance.

PG Production gain.

PL Production loss.

Manuscript received July 17, 2014; revised December 27, 2014, May 09, 2015, September 28, 2015; accepted October 16, 2015. This work was supported in part by the Natural Sciences and Engineering Research Council of Canada and the Vietnam International Education Development. Associate Editor: L. Cui.

The authors are with the Department of Mechanical Engineering, University of Alberta, Edmonton, AB T6G 2G8, Canada (e-mail cuong.dao@ualberta.ca; ming.zuo@ualberta.ca).

Color versions of one or more of the figures in this paper are available online at http://ieeexplore.ieee.org.

Digital Object Identifier 10.1109/TR.2015.2494689

\section{NOTATION}

$N \quad$ Number of components in the system.

$i, j \quad$ Component indices, $i, j \in\{1,2, \ldots, N\}$.

$k, l \quad$ State indices.

$S \quad$ Set of possible states of a component $S$ $=\{0,1,2, \ldots, K\}$.

$s_{i}(t) \quad$ Random variable representing the state of component $i$ at time $t, s_{i}(t) \in S$.

$G \quad$ Set of discrete performance rates in ascending order, $G=\left\{g_{0}, \ldots, g_{K}\right\}$.

$g_{i}(t) \quad$ Random variable representing the instantaneous performance rate of component $i$ at time $t, g_{i}(t) \in G=\left\{g_{0}, \ldots, g_{K}\right\}$.

$\phi(t) \quad$ State of the system at time $t$.

$G(t) \quad$ Random variable representing the instantaneous performance rate of the system at time $t$.

$\lambda_{i ; k} \quad$ Transition rate from state $k$ to state $k-1$ of component $i$ when it degrades due to its local degradation process.

$p_{i, k}(t) \quad$ Probability that an independent component $i$ is in state $k$ at time $t$.

$p_{i, k}^{I}(t) \quad$ Probability that a type 1 s-dependent component $i$ is in state $k$ at time $t$ considering both local degradation and induced failure processes.

$S^{\mathrm{IF}} \quad$ Set of components which can cause immediate failures (IF) of other components.

$S_{i}^{\mathrm{IF}} \quad$ Set of affected components which fails due to induced failure from influencing component $i$.

$\lambda_{i}^{\mathrm{IF}} \quad$ Induced hazard rate of influencing component $i$ which causes type $1 \mathrm{~s}$-dependence to other components, $i \in S^{\mathrm{IF}}$.

$T_{i} \quad$ Time to the IF failure of component $i$.

$\lambda_{i \cdot k}^{(d)} \quad$ Dependent transition rate of component $i$ when it is subjected to type-2 s-dependence.

$\varphi_{i}(\cdot) \quad$ Transition rate dependent function of component $i$ when it is subjected to type-2 s-dependence.

F $\quad$ Complete failure state of the system. 
$P_{k, l}(t) \quad$ Probability a subsystem containing of 2 components is in state $(k, l)$ at time $t$, i.e., component 1 in state $k$ and component 2 in state $l$.

$Q_{k}(t) \quad$ Probability that the system is in state $k$ at time $t$ without considering IF.

$P_{k}(t) \quad$ Probability that the system is in state $k$ at time $t$.

$\mathbf{P}(t) \quad$ Vector of the system state

probabilities in the form $\mathbf{P}(t)=$

$\left[P_{K, K, \ldots, K}(t), P_{K, \ldots, K, K-1}(t), P_{K, \ldots, K, K-1, K-1}(t)\right.$, $\left.\ldots, P_{1,1, \ldots, 1}(t), P_{F}(t)\right]$.

$\Lambda \quad$ Transition rate matrix.

$Y_{i} \quad$ State of component $i$ at the end of the previous mission.

$X_{i} \quad$ State of component $i$ at the end of the maintenance period.

$\tau \quad$ Operating mission duration.

$t_{i}\left(Y_{i}, X_{i}\right)$ Required time for repairing component $i$ from state $Y_{i}$ to state $X_{i}$.

$T$ Total completion time of all selective maintenance activities.

$T_{0} \quad$ Break duration (available time for selective maintenance activities).

$C \quad$ Total cost of the system.

$C_{\mathrm{PG}} \quad$ System production profit/gain.

$c_{g} \quad$ Average profit/gain per unit performance rate when the system satisfies demand.

$C_{\mathrm{PL}} \quad$ System production loss due to missing demand.

$c_{l} \quad$ Average cost loss per unit of missing demand.

$C_{M} \quad$ System maintenance cost in the maintenance break.

$c_{i}\left(Y_{i}, X_{i}\right)$ Cost of repairing component $i$ from state $Y_{i}$ to state $X_{i}$.

$C_{0} \quad$ Available maintenance budget in the break.

$D \quad$ Required demand level.

$R_{s}(\tau, D)$ System reliability at demand level $D$, i.e., probability of the MSS performance is greater than or equal to $D, \operatorname{Pr}(G(t) \geq D)$.

$R_{0} \quad$ Required probability that the MSS operates satisfying the demand in the next mission.

$\delta\left(g_{k}, D\right)$ Unity function, $\delta\left(g_{k}, D\right)=\left\{\begin{array}{l}0 \text { if } g_{k}<D \\ 1 \text { otherwise }\end{array}\right.$.

\section{INTRODUCTION}

T ODAY, maintenance plays an important role in engineering asset management. Researchers have developed various maintenance models for repairable systems in the literature [1]-[3]. In these reported studies, optimum repair, replacement, preventive maintenance, and inspection policies for repairable systems were investigated. However, the majority of these maintenance models ignore the limitation of resources to perform maintenance actions. Many systems have to perform several missions with limited resources, such as time and budget, for maintenance between successive missions. It is often impossible to do all desirable maintenance actions within the available resources. Thus, the maintenance manager has to decide which components to maintain and how to perform maintenance in order to meet the requirements on system's performance. This problem is called selective maintenance.

Selective maintenance was first introduced by Rice et al. [4]. A binary-state series-parallel system with independent and identically distributed components was studied. The probabilities of successfully completing a mission of components are assumed to be known. At the maintenance depot, there are only two available maintenance options on a failed component: replace or do nothing. A non-linear binary integer optimization model was developed to maximize the system reliability in the next mission. Cassady et al. [5] extended the work of Rice et al. by considering components' lifetimes following the Weibull distribution and the possibility of preventive replacement of functioning components. Maillart et al. [6] investigated selective maintenance optimization for the system in [4] that works under $N$ identical missions. Zhu et al. [7] extended the selective maintenance model by considering both the costs of maintenance and production losses for manufacturing lines. Pandey et al. [8] and Liu and Huang [9] studied selective maintenance with imperfect maintenance, i.e., the condition of components after maintenance depends on the cost spent, and the maintenance may bring a component to a state between "as bad as old" and "as good as new." Maaroufi et al. [10] studied selective maintenance for binary systems with S-dependent components where the failure of a component can cause other components to fail immediately. They used the fault tree method to analyze the dependence between components.

In all of these selective maintenance studies, the components are put under the binary assumption, i.e., the components in the system can be in two possible states of functioning or failed. However, in practice, many components can degrade and operate in reduced performance levels. As the component deteriorates, its performance may be in several states, varying from perfect functioning to complete failure. Such a component is called a multistate component, and a system consisting of multistate components is called a multistate system (MSS). The generic concepts and reliability analysis for MSS have been explored in [11] and [12]. In selective maintenance for multistate systems, multiple intermediate repair actions on a component are possible from do-nothing to replacement. Chen et al. [13] modeled selective maintenance for MSS where the probability distributions of multistate components are assumed to be deterministic. The objective of the selective maintenance model is to minimize the cost of maintenance subject to reliability constraints. The shortest path method is used to solve the problem. Pandey et al. [14] studied selective maintenance for multistate series-parallel systems with exponential transition time between states of multistate components. Differential evolution algorithm is used to solve the problem. Recently, Dao et al. [15] extended the model 
in [13] to the case in which the repairs of multistate components are not independent from each other. In this paper, they suggested that repairing multiple components simultaneously is more economical due to the share of setting up, tools, materials and labors.

The assumption of stochastic independence between components is frequently made in selective maintenance studies of multicomponent systems. However, in a specific system design, the current health condition of a component may affect the performance of other components, and the stochastic dependence (S-dependence) exists in most real and complex systems. As stated in [16], the system reliability may be underestimated or overestimated when there is existence of S-dependence, and thus maintenance for those systems needs to be thoroughly investigated. In the literature, there is only one study of selective maintenance for systems with S-dependent components [10], in which the components are binary and the failure of a component can cause other selected components to fail immediately. In [10], the discussion on the effects of the S-dependence on selective maintenance decision making was not investigated. In addition, the dependence relationship in [10] is a special type of S-dependence (type 1), which will be reviewed in the remaining part of this section.

In binary systems, S-dependence is often referred to as "failure dependence" or "failure interaction". In [17], failure interaction in multicomponent systems is classified into two types, i.e., when a component fails: 1) it may cause immediate failure to the other component (type 1) or 2) it may cause damage by increasing the deterioration rate of the affected component (type 2).

In type-1 S-dependence, a component may fail and cause the other component to fail with probability $p$, or leave no effect on the other component with probability $1-p$. Many papers have provided maintenance analysis of systems with type-1 S-dependence, including component replacement policy [18]-[20] and block replacement policy [21]. Regarding type-2 S-dependence, Barros et al. [22] studied a two-unit parallel system where the failure of one component modifies the deterioration rate of the remaining component. A preventive replacement model was given to find the time of maintenance, $T^{*}$, that minimizes the failure and maintenance costs of the system. Lai and Chen [23] studied a two-unit series system where the failure of unit 1 causes damage to unit 2 by increasing the failure rate of unit 2 by a certain amount, while the failure of unit 2 will cause the system immediate failure. Sung et al. [24] extended the two-unit system in [23] by introducing an external shock affected to both components. Albin and Chao [25] studied an $N$-components system in series where the deterioration of the first component changes the failure rate of all $N-1$ subsequent components. However, they did not discuss in details how the failure rate is affected. Rasmekonen and Parlikad [26] considered a system consisting of $M$ parallel non-critical components feeding a critical component. Parallel elements are independent but the performance of the critical components decreases as a parallel component fails. Optimal preventive maintenance is determined to maximize the performance of the critical components.

Recently, more efforts have been made on the S-dependence exhibits in MSS. However, the researchers so far have only fo- cused on the reliability analysis of MSS with S-dependence. In [27] and [28], type-1 S-dependence is explored in MSS where the complete failure originating from a multistate component immediately causes complete failures of other dependent components. In Levitin [29], the universal generating function approach is shown to be efficient to evaluate the reliability of MSS when the conditional probability distributions of the dependent components are deterministic and explicitly known. Dao and Zuo [30] gave preliminary discussions on two types of S-dependence and reliability analysis of the MSS when a multistate component changes its state.

The discussion on S-dependence between multistate components is still limited; only type-1 S-dependence has been considered for multistate systems. In addition, existing maintenance models for binary systems with type-2 S-dependent components are restricted in two-component systems or systems with two stages of dependent components. Thus, our first objective is to consider both types of S-dependence in an $N$-component multistate series system. An approach based on the stochastic process will be used to evaluate the system reliability. Secondly, existing papers in selective maintenance have not taken both the costs and the profit associated with the MSS performance rates into consideration. Both the production loss and gain corresponding to multiple working levels of the system and the maintenance costs are integrated into the selective maintenance cost model. To sum up, a cost-based selective maintenance model will be developed for multistate systems with two types of S-dependence. The objective of the maintenance model is to maximize the total system profit considering resources and reliability constraints. Examples and results show that ignoring S-dependence leads to an optimistic estimation of the system reliability and profit for the illustrative case. The proposed selective maintenance model is expected to help the maintenance decision maker find the most economical maintenance strategy under reliability and resource constraints.

The remainder of this paper is organized as follows. Section II describes the MSS with two types of dependence. A method of reliability analysis for the system with S-dependent components is presented in Section III. Selective maintenance policies and a cost-based maintenance optimization model are developed in Section IV. Illustrative examples, results and discussion will be presented in Section V, and a general conclusion is provided in Section VI.

\section{Multistate Systems With S-DePendent Components}

The multistate system in this study consists of $N$ multistate components connected in series (Fig. 1). Each multistate component $i$ can be in one state in a set of $K+1$ possible discrete states, $S=\{0,1,2, \ldots, K\}$, associated with a corresponding working performance rate in a set of $G=\left\{g_{0}, g_{1}, \ldots, g_{K}\right\}$. $K$ is the perfect functioning state, 0 is the complete failure state, and other intermediate states, $1,2, \ldots, K-1$, are imperfect functioning states, i.e., the component can continue operating but at a reduced performance rate. The set of state and performance rates are in ascending order, i.e., $g_{k}<g_{l}$ if $k<l$.

At time $t$, the state of component $i$, denoted by $s_{i}(t)$, is a random variable, $s_{i}(t) \in S=\{0,1,2, \ldots, K\}$. The perfor- 


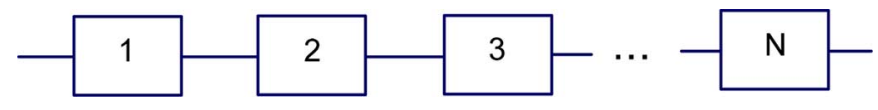

Fig. 1. Series system.

mance rate of component $i$ is denoted by $g_{i}(t)$ and the probability that component $i$ is in state $k$ at time $t$ is defined as in

$$
p_{i, k}(t)=\operatorname{Pr}\left\{g_{i}(t)=g_{k}\right\} .
$$

The system performance rate $G(t)$ is defined as minimum performance rate of its components as in

$$
G(t)=\operatorname{Min}\left\{g_{1}(t), g_{2}(t), \ldots, g_{N}(t)\right\}
$$

The system's state associated with performance rate $G(t)$ at time $t$ is $\phi(t)$, given by

$$
\phi(t)=\operatorname{Min}\left\{s_{1}(t), s_{2}(t), \ldots, s_{N}(t)\right\}
$$

The state of the system and its performance rate can be determined by the combination of its components' states from (2) and (3). The state probability of the system is

$$
P_{k}(t)=\operatorname{Pr}\{\phi(t)=k\}=\operatorname{Pr}\left\{G(t)=g_{k}\right\} .
$$

In the presented MSS, there might be two types of dependence when a multistate component changes its state as follows.

- Type 1-Immediate failure dependence (IFD): when a component (influencing component) fails, it may cause immediate failures of some other affected components. For example, a component fails and creates fire that causes some nearby components to fail immediately; or an electrical component fails and causes a voltage spike that triggers the failure of some other components in the system that do not have protective device.

- Type 2-Gradual degradation dependence (GDD): as the multistate component deteriorates with time and once it degrades to a lower working performance rate level, it does not cause affected components to fail immediately but results in increasing or decreasing the degradation of the affected components. In our model, when a component degrades to a lower state, i.e., its output performance rate decreases, both the state and the degradation rate of the next component in the series system are affected. This dependence mechanism occurs when the components have direct connection to each other and the output of a component controls the performance of the affected component. A system consisting of a water feed pump station and a steam turbine can serve as an illustrative example. This is a two-component series system by considering the water feed pump station as component 1 and the turbine as component 2 . When the pump station works at its maximum performance rate of $x$ (ton/h), the healthy turbine can generate its maximum power corresponding to $x$. When the pump's health condition deteriorates, the amount of water per unit time delivered to the next component (the turbine) decreases. This will result in a decrease in the degradation

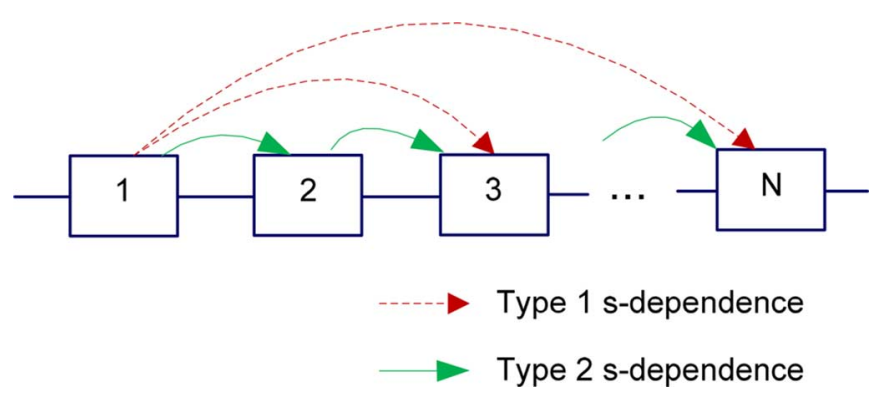

Fig. 2. Two types of dependence in a multistate series system.

rate of the turbine due to the reduced load that the turbine needs to handle, and for the same reason, the output performance of the turbine decreases too. Thus, in this paper, we assume that both the performance state and the degradation rate of a component may be affected by the degradation of the influencing component.

The two types of dependence in multistate series systems can be represented in Fig. 2.

In multistate systems, it is seen that type $1 \mathrm{~S}$-dependence can be defined in the same way as in binary systems, i.e., the failure originating from a multistate component can immediately cause complete failures of other affected components. In Fig. 2, the red dashed arrows represent type-1 S-dependence relationship, e.g., the failure of component 1 can cause immediate failure (IF) to component 3 and component $N$. As stated in [18], a component may cause type-1 S-dependence failure with probability $p$, or leave no effect on the other components with probability $1-p$. It is assumed that the influencing component can fail in two failure processes: one is its local degradation process that has no effect on other components, and the other failure process that causes induced failures to affected components.

On the other hand, type-2 S-dependence in multistate context describes the "degradation dependence" of a multistate component on the degradation of other components in the system. The transition rate between states of an affected component varies depending on the instantaneous performance rate of the influencing components. In MSS, the performance rate is often defined as productivity or capacity. In series systems, the input of a component is the output performance rate of its predecessor and the output performance rate of a component is the input of its subsequent component. Thus, type-2 S-dependence has a chain effect (green arrows in Fig. 2). It is also recognized that a component $i$ is actually affected by the output performance rate of the group of components from $1,2, \ldots, i-1$, not just from a single component $i-1$. In other words, the instantaneous performance rate of component 1 influences the degradation of component 2; the performance rate of the group of components 1 and 2 influences the degradation of component 3 and so forth (see the green-solid arrows in Fig. 2). Thus, a component $i, i=2,3, \ldots, N-1$, can be both an influencing component to its subsequence components and an affected component from its predecessors.

In summary, the MSS with S-dependent components has the following characteristics.

- The system consists of $N$ components connected in series. 


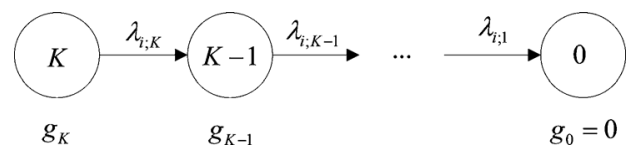

Fig. 3. Transition diagram of a MS component without S-dependence.

- The components can operate in one of $K+1$ states with the performance rates ranging from perfect functioning to complete failure.

- All components in the system degrade due to their local degradation process. Some components can fail in an induced failure mode that causes immediate failures of affected components (type-1 S-dependence). Once an affected component fails, it cannot cause IF to any other component in the system.

- Components may degrade to a lower working performance rate and it affects the degradation process of subsequent components by increasing or decreasing the degradation rate of the subsequent components (type-2 S-dependence).

- A component can cause both types of S-dependence and a component can be subjected to both types of S-dependence.

\section{Multistate System Reliability Analysis}

\section{A. Multistate Systems With Independent Components}

All components in the system degrade due to a local degradation process from the perfect functioning state to complete failure state. Without considering S-dependence, the probabilities associated with different states of a component $i$ at time $t$ due to its local degradation process can be represented by a set

$$
\mathbf{p}_{i}(t)=\left\{p_{i, 0}(t), p_{i, 1}(t), p_{i, 2}(t), \ldots, p_{i, K}(t)\right\}
$$

Fig. 3 shows the transition diagram that represents the degradation of a multistate component without considering S-dependence.

In Fig. 3, component $i$ has $K+1$ possible states from perfect functioning (state $K$ ) to complete failure (state 0 ) with nominal transition rate from state $k$ to state $k-1$ of $\lambda_{i ; k}$. For simplicity, we assume that the sojourn time at each state of the component follows exponential distributions, and the component degrades gradually, i.e., if the component is currently in state $k$, it will degrade to state $k-1$ before reaching a worse state $k$ -2 . With these assumptions, the next transition of a component only depends on its current state, and the stochastic process governing the local degradation process is a Markov chain. The state probabilities can be determined by solving the Chapman-Kolmogorov (C-K) system of differential equations as follows:

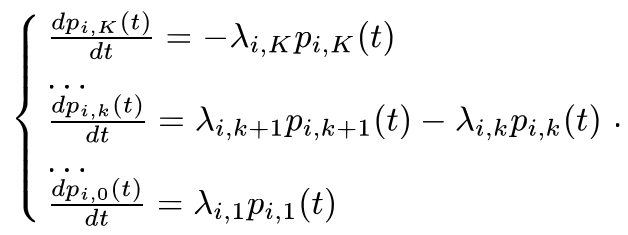

The last equation based on transition diagram is $\left(d p_{i, 0}(t)\right) /(d t)=\lambda_{i, 1} p_{i, 1}(t)$. However, it can be replaced by $\sum_{k=0}^{K} p_{i, k}(t)=1$ since the sum of all state probabilities of a component is always equal to 1 . Further discussions on modeling the degradation of multistate independent components can be found in [31]. Regarding the method to solve the system of equations, there are several methods including numerical and analytical methods. In this paper, we solve the system of equations using a method described in [32] to solve it analytically.

\section{B. Modeling Two Types of S-Dependence of Components}

1) Type-1 S-Dependence: In the system, we denote $S^{\mathrm{IF}}$ as the set of components that can fail and cause immediate failures (IF) of other components and $S_{i}^{\mathrm{IF}}$ as the set of components affected by the induced failure of component $i, i \in S^{\mathrm{IF}}$. When component $i$ fails, it will either cause all the affected components in $S_{i}^{\mathrm{IF}}$ to fail with probability $p$, or leave no effect on these components with probability $1-p$. As a result, an influencing component $i$ may fail following two failure processes: local failure and induced failure. The first failure process is the local degradation process that does not affect any other components as described in Section III-A. The induced failure process causes the affected components to fail immediately. In previous studies, the probability $p$ is assumed to be a predetermined value, but, in this paper, the probability of induced failure from component $i, p_{i}^{\mathrm{IF}}(t)$, is defined as a function of time:

$$
p=p_{i}^{\mathrm{IF}}(t)=1-\exp \left\{-\int_{0}^{t} \lambda_{i}^{\mathrm{IF}}(s) d s\right\}
$$

where $\lambda_{i}^{\mathrm{IF}}(t)$ is the induced failure hazard rate of component $i$. In this case, a state $0^{\prime}$ is added into the set of states of the influencing component to represent the induced failure state related to type-1 S-dependence. The performance rates of influencing and affected components at state $0^{\prime}$ are $g_{0^{\prime}}=0$. The state probability vector of the influencing component $i$ takes the following form:

$$
\mathbf{p}_{i}(t)=\left\{p_{i, 0^{\prime}}^{I}(t), p_{i, 0}^{I}(t), p_{i, 1}^{I}(t), \ldots, p_{i, K}^{I}(t)\right\} .
$$

In (8), $p_{i, 0^{\prime}}^{I}(t)$ is the probability that the influencing component $i$ fails and causes IF. Thus, $p_{i, 0^{\prime}}^{I}(t)=p_{i}^{\mathrm{IF}}(t)$ and $p_{i, k}^{I}(t), k=$ $0,1, \ldots, K$ is the state probability of component $i$ in the case that it does not fail due to induced failure process. We assume that the local and induced failure processes are stochastically independent, then

$$
p_{i, k}^{I}(t)=p_{i, k}(t) \exp \left\{-\int_{0}^{t} \lambda_{i}^{\mathrm{IF}}(s) d s\right\}
$$

where $p_{i, k}(t)$ is the state probability obtained by its local degradation process which can be obtained from solving the system of (6).

The state probability distribution of an affected component $j$ $\in S_{i}^{\mathrm{IF}}$ depends on the state of component $i$. In this paper, we assume that an affected component $j$ cannot cause immediate failure to any other component in the system. This also means that a component in the set of influencing components, $S^{\mathrm{IF}}$, cannot be affected by another component in the same set $S^{\mathrm{IF}}$. 
If the induced failure does not occur, the state probability vector of component $j$ is $\mathbf{p}_{j}(t)=$ $\left\{p_{j, 0}(t), p_{j, 1}(t), \ldots, p_{j, K}(t)\right\}$, where $p_{j, k}(t), k=0,1, \ldots, K$ is the state probability of component $j$ obtained by solving (6). If the induced failure of component $i$ occurs, the state of component $j$ is 0 , i.e., $\mathbf{p}_{j}(t)=\{1,0, \ldots, 0\}$. Thus, we have the conditional probability distribution of component $j$ as shown by (10), at the bottom of the page. There is another way to explain (9) and (10) in conditional probabilities by defining $\left\{s_{i}(t), t \geq 0\right\}$ with a state space of $\{0,1, \ldots, K\}$ as the stochastic process governing the local degradation process. Another stochastic process $\left\{s_{i}^{I}(t), t \geq 0\right\}$ with a state space $\left\{0^{\prime}, 0,1, \ldots, K\right\}$ is defined to represent type-1 S-dependence of influencing component $i$ as follows:

$$
s_{i}^{I}(t)= \begin{cases}s_{i}(t), & \text { if } t<T_{i} \\ 0, & \text { if } T_{i} \leq t\end{cases}
$$

where $T_{i}$ is the time to IF of component $i$.

From these formulations, for $0 \leq k \leq K$, we have

$$
\begin{aligned}
& \operatorname{Pr}\left\{s_{i}^{I}(t)=k \mid T_{i}>t\right\}=\operatorname{Pr}\left\{s_{i}(t)=k \mid T_{i}>t\right\} \\
& \quad=\operatorname{Pr}\left\{s_{i}(t)=k\right\}=p_{i, k}(t) \\
& \operatorname{Pr}\left\{s_{i}^{I}(t)=k \mid T_{i} \leq t\right\}=0 \\
& \Rightarrow \operatorname{Pr}\left\{s_{i}^{I}(t)=k\right\}=\operatorname{Pr}\left\{s_{i}(t)=k \mid T_{i}>t\right\} \operatorname{Pr}\left\{T_{i}>t\right\} \\
& \quad=p_{i, k}(t) \exp \left\{-\int_{0}^{t} \lambda_{i}^{\mathrm{IF}}(s) d s\right\} .
\end{aligned}
$$

Equation (13) is the same as (9).

When $k=0^{\prime}$, we have

$$
\begin{aligned}
& \operatorname{Pr}\left\{s_{i}^{I}(t)=0^{\prime} \mid T_{i} \leq t\right\}=\operatorname{Pr}\left\{T_{i} \leq t \mid T_{i} \leq t\right\}=1 \\
& \operatorname{Pr}\left\{s_{i}^{I}(t)=0^{\prime} \mid T_{i}>t\right\}=0 .
\end{aligned}
$$

Similarly, we can define a stochastic process $\left\{s_{j}^{A}(t), t \geq 0\right\}$ with state space $\{0,1, \ldots, K\}$ to represent the type- 1 S-dependence for an affected component $j$ as given by (15), shown at the bottom of the page. This formula (15) is equivalent to (10).

2) Type-2 S-Dependence: Type-2 S-dependence exists when components have direct connection to each other and the output of the influencing components controls the performance of the affected components. In series systems, a component $i, i=$ $2,3, \ldots, N-1$, can be both an influencing component to its subsequent component and an affected component from its predecessor. We assume that the degradation of component $i, i>1$ depends on the output performance rate of its preceding component $i-1$. When its predecessor $i-1$ is at the perfect functioning state, i.e., $g_{i-1}(t)=g_{K}$, component $i$ degrades at its nominal transition rate, i.e., the transition rate of the local degradation process $\lambda_{i ; k}$. When component $i-1$ degrades and $g_{i-1}(t)<g_{K}$, type 2 S-dependence between component $i$ and its immediate predecessor is described as follows.

- The performance rate dependence: the performance rate of component $i$ is automatically adjusted to the output of performance rate supply to it, i.e., $g_{i}(t) \leq g_{i-1}(t)<g_{K}$

- The degradation rate dependence: the transition rate between states of the affected component $i, \lambda_{i ; k}^{(d)}$, also depends on $g_{i-1}(t)$. We use $\varphi_{i}(\cdot)$ as a dependent function to describe type-2 S-dependence of component $i$ on its immediate predecessor as follows:

$$
\begin{aligned}
\lambda_{i ; k}^{(d)}\left(g_{i-1}(t)\right) & =\varphi_{i}\left(g_{i-1}(t)\right) \lambda_{i ; k} \\
& =\left(\frac{g_{i-1}(t)}{g_{K}}\right)^{m_{i}} \lambda_{i ; k}, \quad k=1,2, \ldots, K
\end{aligned}
$$

In (16), $\varphi_{i}(\cdot)$ is a function of the instantaneous performance rate of the component $i-1, g_{i-1}(t)$, and a dependent exponent $m_{i} . m_{i}$ is an exponent that characterizes the degree of dependence between component $i$ and its immediate predecessor. When $m_{i}>0$, the reduced performance of the influencing component decreases the degradation process of the affected component, i.e., $\lambda_{i: k}^{(d)}<\lambda_{i ; k}$; when $m_{i}<0$, the reduced performance of the influencing component increases the degradation process of the affected component, i.e., $\lambda_{i ; k}^{(d)}>\lambda_{i ; k}$. When $m_{i}=0$, we have $\lambda_{i ; k}^{(d)}=\lambda_{i ; k}$, i.e., the transition rate doesn't change but the performance of the affected component still depends on the performance of its preceding component.

In this paper, we use the power law with $m_{i}$ being an exponent representing the degree of dependence of component $i$ on its preceding component. In general, the transition rate of an affected component may also depend on state $k$. If this is the case, the component type and state effect can be characterized by an exponent $m_{i, k}$, where $m_{i, k}$ represents the dependence of component $i$ at state $k$ on its preceding component. In this paper, we assume that the performance rate associated with each state of a component is uniformly distributed and the dependence effects are equal for each state, i.e., $m_{i, k}$ can be considered as $m_{i}$ for all $k$. In addition, the same methods of reliability analysis and maintenance optimization in the next sections can be applied for either case where $m_{i}$ or $m_{i, k}$ is used.

$$
\mathbf{p}_{j}(t)= \begin{cases}\left\{p_{j, 0}(t), p_{j, 1}(t), \ldots, p_{j, K}(t)\right\}, & \text { if no IF occurs } \\ \{1,0, \ldots, 0\}, & \text { if IF from at least one influencing component occurs }\end{cases}
$$

$$
s_{j}^{A}(t)= \begin{cases}s_{j}(t), & \text { if } s_{i}^{I}(t) \neq 0^{\prime}, \text { for every } i \in S^{\mathrm{IF}} \text { such that } j \in S_{i}^{\mathrm{IF}} \\ 0, & \text { if } s_{i}^{I}(t)=0^{\prime}, \text { for some } i \in S^{\mathrm{IF}} \text { such that } j \in S_{i}^{\mathrm{IF}}\end{cases}
$$



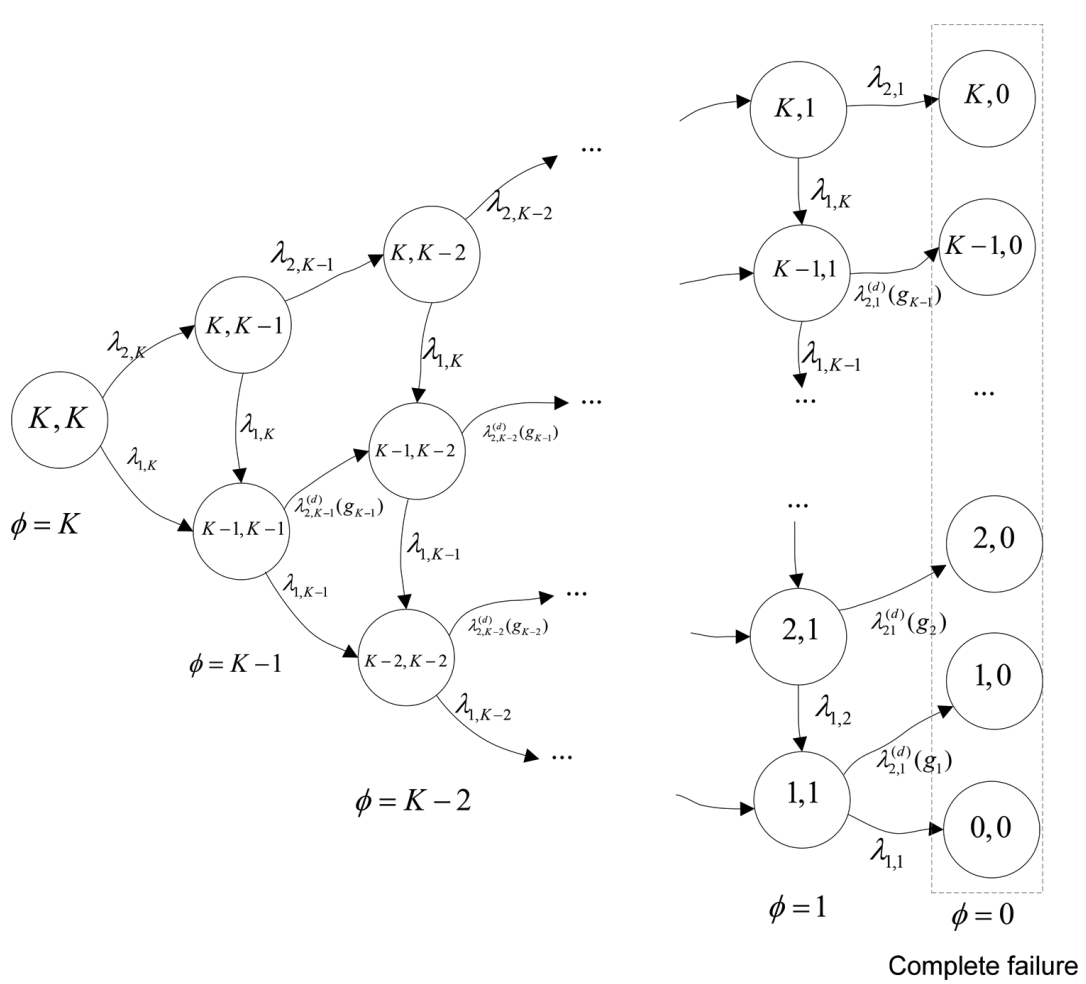

Fig. 4. Transition diagram of two components with type-2 S-dependence.

\section{Reliability Analysis of MSS With Dependent Components}

1) Markov Analysis for Components With Type 2 s-Dependence: In type-2 S-dependence, we see that the degradation of a component $i, i>1$, depends not only on its current state but also on the state of its immediate predecessor. If we consider simultaneously the state of component $i$ and the state of its immediate predecessor at the same time, the degradation of the subsystem of components $i-1$ and $i$ can be modeled as a Markov chain.

To illustrate the type-2 S-dependence as a Markov chain, we take an example of two components connected in series, wherein each component's state space is $\{0,1,2, \ldots, K\}$. Although the system state ismin $\left\{s_{1}, s_{2}\right\} \in\{0,1, \ldots, K\}$, we denote the system state as a pair of $\left(s_{1}, s_{2}\right)$. The system state space is $\{(K, K),(K, K-1), \ldots,(1,0),(0,0)\}$. With this notation, the system states denoted by $(k, k-1)$ and $(k-1, k-1)$ are considered to be different although the output performance of the system is identical. The first element in system state $\left(s_{1}, s_{2}\right)$ determines the state and the associated output performance rate of component 1 . The second element in the pair $\left(s_{1}, s_{2}\right)$ depends on the value of the first element. However, the stochastic process utilizing the pair notation is a Markov chain since the next state of the system in pair notation $\left(s_{1}, s_{2}\right)$ only depends on its current state. The stochastic process with the local degradation process is $\left\{\left(s_{1}(t), s_{2}(t)\right)\right\}$ with transition rates $\left(\lambda_{1, k}, \lambda_{2, l}\right)$. When type- 2 dependence is introduced, the stochastic process is modified to $\left\{\left(s_{1}(t), s_{2}(t)\right)\right\}$ with additional requirements of $s_{1}(t) \geq s_{2}(t)$ and the transition rates of $\left(\lambda_{1, k}, \lambda_{2, l}^{(d)}\right), k, l \in$ $\{1,2, \ldots, K\} ; l \leq k$ to represent type-2 S-dependence between the two components as described in Section III-B. The state transition diagram for the group of two components is shown in Fig. 4.
In Fig. 4, type-2 S-dependence happens whenever the influencing component changes its state, which may result in state dependence and transition rate dependence. Transition rate dependence always happens when the influencing component changes its state, but state dependence occurs only if the transition of the influencing component causes its state to be less than the state of the affected component. For example, there are two transition paths from state $(K, K)$ to state $(K-1, K$ $-1)$, which are $(K, K) \rightarrow(K, K-1) \rightarrow(K-1, K-1)$, and $(K, K) \rightarrow(K-1, K-1)$. In the first transition path, we can understand that there is no dependence in the first transition $(K, K) \rightarrow(K, K-1)$. In the system state $(K, K-1)$, the second component has its nominal transition rate $\lambda_{2, K-1}$. From this system state, when component 1 degrades from state $K$ to $K-1$, i.e., the system state becomes $(K-1, K-1)$, the transition rate of component 2 is modified to $\lambda_{2, K-1}^{d}\left(g_{K-1}\right)$ since the performance rate of component 1 has changed. Although state dependence does not take place, type $2 \mathrm{~S}$-dependence still occurs in this transition in terms of transition rate dependence. In the second path, there is no transition from the state $(K, K)$ to state $(K-1, K)$, because when component 1 enters state $K$ -1 the state of component 2 is instantly adjusted to $K-1$ and its transition rate is also adjusted to $\lambda_{2, K-1}^{d}\left(g_{K-1}\right)$ due to type $2 s$-dependence. It is noted that state dependence only happens in the second path. This is because state dependence happens if the transition causes the state of the affected component to be greater than the state of the influencing component.

When any component degrades to state " 0 ", the system is in complete failure state. In Fig. 4, we can combine all such states with at least one component in state " 0 " as the " $F$ " state or complete failure state. " $F$ " is an absorbing state during the mission, i.e., no transition can happen further when the system is 
failed. The $\mathrm{C}-\mathrm{K}$ system of equations for type-2 S-dependence is written as in (17), shown at the bottom of the page. In (17), the last equation can be replaced by $\sum_{k=1}^{K} \sum_{l=1}^{k} P_{k, l}(t)+P_{F}(t)=1$ since the sum of all system state probabilities is 1 . In the transition diagram, it is noted that there are always $N$ "going-out" transitions for each state (except absorbing state " $F$ ") representing the degradation of each of the two components in the system. In general, there are also $N$ "going-in" transitions for each state (except absorbing state " $F$ " and the state at which the influencing component is in perfect functioning state $K$ ) representing the degradation of components from higher states.

The system of $\mathrm{C}-\mathrm{K}$ equations can be rewritten in matrix form [32]

$$
\frac{d \mathbf{P}(t)}{d t}=\mathbf{P}(t) \Lambda
$$

In (17), there are a total of $f$ equations, $f=$ number of state combinations of all components in the system. Then, $\mathbf{P}(t)$ is a row vector, $1 \times f$, and $\Lambda$ is the transition rate matrix, $f \times f$. For system of (18), we can write matrix $\Lambda$ as given by (19), shown at the bottom of the page. It is noted that the sum of all elements in a column of matrix $\Lambda$ is always equal to zero. Given $\mathbf{P}(0)$ as a row vector of initial condition, we can solve the system of $\mathrm{C}-\mathrm{K}$ equations and determine the system state probabilities. The solutions to the system of equation (18) take the following form [32].

$$
\mathbf{P}(t)=\mathbf{P}(0) \cdot V \cdot \mathrm{e}^{D t} \cdot V^{-1}
$$

where $V$ is a matrix of eigenvectors of matrix $\Lambda(f \times f) ; D$ is a diagonal matrix with diagonal elements being eigenvalues of $\Lambda$.

When the system has more than two components, the components' states are grouped and denoted in a similar way. Each system state has to be denoted by $N$-tuple for an $N$-component series system. Markov properties and analysis for the system can be carried out similarly. The degradation of component $i$ can be explicitly determined when the current states of the group of components $\{1,2, \ldots, i-1\}$ are included in the system state.
The next state of the system denoted by $\left\{s_{1}, s_{2}, \ldots, s_{N}\right\}$ only depends on its current state. The state transition diagram and the system of C-K equations can be obtained similarly.

In this paper, the state space of the Markov model depends on the number of components and the number of health states of each component. The complexity of the Markov model depends on two factors: 1) the system state space and 2) the method used to solve the system of $\mathrm{C}-\mathrm{K}$ equations. The complexity of the method used to solve the C-K equation by [32] is not our focus in this paper. Here, we will discuss the state space of the system model and illustrate the complexity of the system model.

Consider a system of $N$ multistate components with type-2 S-dependence. Each component may be in $K+1$ possible states. We use $f_{N}^{K}, N \geq 1, K \geq 0$, to represent the number of states of such a system with $N$ components and $K$ is the common maximum state of each component.

- When $N=1$, i.e., the system has only one component, and we have $f_{1}^{K}=K+1$.

- When $N=2$, the state dependence requirement dictates that $s_{1} \geq s_{2}$ where $\mathrm{s}_{1}$ is the state of component 1 and $\mathrm{s}_{2}$ is the state of component 2 . Component 1 is the influencing component while component 2 is the affected component. Considering $\mathrm{s}_{1}$ may take integer values from 0 to $K$, inclusive and $\mathrm{s}_{2}$ may take integer values from 0 to $s_{1}$, inclusive, the number of states of the system in $\left(s_{1}, s_{2}\right)$ is then equal to $f_{2}^{K}=f_{1}^{0}+f_{1}^{1}+\cdots+f_{1}^{K-1}+f_{1}^{K}=((K+1)(K$ $+2)) /(2)$.

- When there are $N$ components in the system, there is a cascading dependence requirement such that $K \geq s_{1} \geq$ $s_{2} \geq \cdots \geq s_{N} \geq 0$. Using similar reasoning, we can find the number of states of the system in $\left(s_{1}, s_{2}, \ldots, s_{N}\right)$ as $f_{N}^{K}=\sum_{k=0}^{K} f_{N-1}^{k}=\prod_{i=1}^{N}((K+i) /(i))$.

We can see that $f_{N}^{K}=\prod_{i=1}^{N}((K) /(i)+1) \gg((K) /(N)+1)^{N}$ and $(K) /(N)+1>1$. Thus, the number of system states increases with the order of at least $\mathrm{O}\left(((K) /(N)+1)^{N}\right)$ when $N$ $\rightarrow \infty$ and $K \rightarrow \infty$. This exponential increase in the number of system states limits the model presented in this paper for systems with large $N$ and $K$ values. In the future, more efficient

$$
\left\{\begin{array}{l}
\frac{d P_{K, K}(t)}{d t}=-\left(\lambda_{1, K}+\lambda_{2, K}\right) P_{K, K}(t) \\
\frac{d P_{K, K-1}(t)}{d t}=\lambda_{2, K} P_{K, K}(t)-\left(\lambda_{2, K-1}+\lambda_{1, K}\right) P_{K, K-1}(t) \\
\frac{d P_{K-1, K-1}(t)}{d t}=\lambda_{1, K} P_{K, K}(t)+\lambda_{1, K} P_{K, K-1}(t)-\left(\lambda_{1, K-1}+\lambda_{2, K-1}^{(d)}\left(g_{K-1}\right)\right) P_{K-1, K-1}(t) \\
\frac{d P_{F}(t)}{d t}=\lambda_{2,1} P_{K, 1}(t)+\lambda_{2,1}^{(d)}\left(g_{K-1}\right) P_{K-1,1}(t)+\cdots+\lambda_{2,1}^{(d)}\left(g_{1}\right) P_{1,1}(t)+\lambda_{1,1} P_{1,1}(t)
\end{array}\right.
$$

$$
\Lambda=\left[\begin{array}{cccccc}
-\left(\lambda_{1, K}+\lambda_{2, K}\right) & 0 & 0 & \cdots & 0 & 0 \\
\lambda_{2, K} & -\left(\lambda_{2, K-1}+\lambda_{1, K}\right) & 0 & \cdots & 0 & 0 \\
\lambda_{1, K} & \lambda_{1, K} & -\left(\lambda_{1, K-1}+\lambda_{2, K-1}^{(d)}\left(g_{K-1}\right)\right) & \cdots & 0 & 0 \\
\cdots & \cdots & \cdots & \cdots & \cdots & \cdots \\
0 & 0 & 0 & \cdots & \lambda_{1,1}+\lambda_{2,1}^{(d)}\left(g_{1}\right) & 0
\end{array}\right]
$$


methods for solving the system model are needed to handle the relationships between components proposed in this paper.

2) Combining Two Types of S-Dependence in System Reliability Analysis: When both types of dependence are considered, the stochastic process governing the degradation of each component cannot be defined individually since there is a chain effect in type-2 S-dependence from the first component to the last component in a series system. Thus, all components in the system are considered at the same time. In fact, we do not need to define the stochastic process for each component individually to determine the system reliability. The system state probabilities can be first obtained using the Markov analysis for the system with type-2 S-dependence as outlined in Section 3.3.1. Then, type-1 S-dependence is incorporated in the event that the system is in a failure state, which is caused when at least one immediate failure occurs.

A stochastic process for the system with $N$ components considering both types of S-dependence is defined as follows. For a system of $N$ components, we use $S^{\mathrm{II}}(t)=$ $\left\{s_{1}^{\mathrm{II}}(t), s_{2}^{\mathrm{II}}(t), \ldots, s_{N}^{\mathrm{II}}(t)\right\}$, which is an $N$-tuple, to represent the system state considering type-2 S-dependence. The superscript refers to type-2 S-dependence and the subscript is the component in the system. Each $N$-tuple, $S^{I I}(t)=\left\{s_{1}^{I I}(t), s_{2}^{I I}(t), \ldots, s_{N}^{I I}(t)\right\}$, exactly defines the state of the system and $\left\{S^{I I}(t), t \geq 0\right\}$ is the stochastic process representing the system degradation due to type 2 $s$-dependence. Then, the stochastic process governing both types of $s$-dependence of the system is $\left\{S_{S}(t), t \geq 0\right\}$, where $S_{S}(t)$ is defined in (21), shown at the bottom of the page.

In the system of (17), the state probabilities of the system are in the form of $P_{s_{1}, s_{2}, \ldots, s_{N}}(t)$. Given the initial state of each component in the system, the system of $\mathrm{C}-\mathrm{K}$ equations can be solved using the method presented in [32] to obtain the state probabilities $P_{s_{1}, s_{2}, \ldots, s_{N}}(t)$. Denote $Q_{k}(t)$ as the probability that the system is in state $k$ at time $t$ without considering IF. We can easily transfer the system state probabilities $P_{s_{1}, s_{2}, \ldots, s_{N}}(t)$ into $Q_{k}(t), k=0,1, \ldots, K$ by applying

$$
Q_{k}(t)=\sum_{\min \left\{s_{i}\right\}=k} P_{s_{1}, s_{2}, \ldots, s_{N}}(t) .
$$

When IF from component $i$ happens, both influencing and affected components are in failed state, and the system is also in failed state. In addition, if a component is in state 0 , IF failure cannot happen to the system. Thus, the state probabilities of the system considering type-1 S-dependence can be represented as follows.

For $k=1,2, \ldots, K$, we have

$$
\begin{aligned}
P_{k}(t) & =Q_{k}(t) \times \operatorname{Pr}\{\text { there is no IF }\} \\
& =Q_{k}(t) \times \prod_{i \in S^{\mathrm{IF}}} p_{i}^{\mathrm{IF}}(t)
\end{aligned}
$$

and

$$
\begin{aligned}
P_{0}(t)= & Q_{0}(t) \times \operatorname{Pr}\{\text { there is no IF }\} \\
& +\operatorname{Pr}\{\text { at least } 1 \text { IF occurs }\} \\
= & Q_{0}(t) \times \prod_{i \in S^{\mathrm{IF}}} p_{i}^{\mathrm{IF}}(t)+\left(1-\prod_{i \in S^{\mathrm{IF}}} p_{i}^{\mathrm{IF}}(t)\right) .
\end{aligned}
$$

From (23) and (24), we can get the system state probabilities $P_{k}(t), k=0,1,2, \ldots, K$. The system reliability is the probability that the system can operate and satisfy a required demand $D$. Once the state probabilities $P_{k}(t)$ are determined, the system reliability can be obtained using a zero-one function, $\delta\left(G_{k}, D\right)$.

$$
R_{s}(t, D)=\operatorname{Pr}\{G(t) \geq D\}=\sum_{k=0}^{K} P_{k}(t) \delta\left(g_{k}, D\right)
$$

where

$$
\delta\left(g_{k}, D\right)= \begin{cases}0, & \text { if } g_{k}<D \\ 1, & \text { otherwise }\end{cases}
$$

\section{Selective Maintenance Model}

Many systems have to work consecutive missions with break intervals between missions. "Mission" is a task that the system has to perform. A mission is associated with a time duration. For example: an aircraft has to perform a mission, i.e., a flight, between two airports, a manufacturing system has to operate in a day shift from 6 am to $6 \mathrm{pm}$. Between two missions, there is a break to do maintenance, e.g., stop time at the airport in the aircraft example, and night time off-working in the manufacturing system example. In our selective maintenance model, there is a maintenance break between two successive missions during which maintenance can be performed. During the next mission, if IF happens or when a component degrades to a state that cannot satisfy demand, the system is considered as failed and the mission is failed. No repair action is allowed within a mission. Thus, the maintenance decision maker must take the probability of successfully completing the next mission, i.e., the system reliability, into consideration prior to the start of the mission. The proposed maintenance model will optimize the maintenance decisions before a mission starts so that the system reliability is greater than or equal to a required threshold.

\section{A. Maintenance Actions on a Multistate Component}

In the selective maintenance problem, one needs to find the optimal combination of maintenance actions on the components when they arrive at the maintenance depot under the limited resources. Denote $Y_{i}$ and $X_{i}$ as the states of component $i$ at the time of entering and exiting the maintenance depot, respectively. It is assumed that the system is inspected at the time of entering the maintenance depot and the components' states are

$$
S_{S}(t)= \begin{cases}S^{I I}(t), & \text { if } s_{i}^{I}(t) \neq 0^{\prime}, \text { for } \forall i \in S^{\mathrm{IF}} \\ 0, & \text { if } s_{i}^{I}(t)=0^{\prime} \text { for at least a component } i \in S^{\mathrm{IF}}\end{cases}
$$


detected instantaneously, i.e., the state vector at the time of entering the maintenance depot is explicitly known. The maintenance actions do not worsen the condition of the component, i.e., $Y_{i} \leq X_{i}$. In general, multiple maintenance options are available on a multistate component when it is in the maintenance depot as follows.

- Do-nothing: the current health state of the component does not change at the maintenance depot, i.e., $X_{i}=Y_{i}$.

- Replacement: The component is renewed to the state "as good as new."

- Imperfect maintenance (IM): $Y_{i}<X_{i}<K$.

In a selective maintenance modeling for the system consisting of multiple S-dependent components, it is necessary to consider the effect of S-dependence on the selection of maintenance activities. In this paper, we take S-dependence between components into account by considering the following aspects when modeling the maintenance activities of components.

- At the maintenance depot, if the influencing component is in induced failure state, the maintenance action of its affected components is valid only when the influencing component is selected, i.e., when the affected component is selected in a maintenance strategy, the influencing component in type-1 S-dependence is also selected.

- Among maintenance actions for type-2 S-dependence components, the repair of the affected components cannot bring it to a state greater than the influencing component's state. Because of the state dependence between components, the repair action to bring the affected component to a state greater than the state of the influencing component requires more maintenance resources but does not improve the system reliability and stochastic degradation in the next mission in comparison with repairing the affected component to the state equal to the state of the influencing component.

\section{B. MSS Profit and Cost Evaluation}

In this paper, our aim is to build a cost-based selective maintenance model to identify the most economical maintenance strategy when S-dependence exists. To do so, we analyze the profit and costs for the system during the maintenance period and in the next operating mission, including:

- the system production gain and loss associated with its performance rate in the next mission.

- the maintenance cost in the maintenance depot.

1) System Production Gain and Loss: In MSS, the performance rate often represents productivity or capacity. Thus, the MSS production gain and loss are evaluated using the multiple output performance rates it can produce in the next mission. Let $c_{g}$ and $c_{l}$ be the average production gain and loss per unit of performance. It is assumed that $c_{g}$ and $c_{l}$ are given. This is a reasonable assumption since the values of $c_{g}$ and $c_{l}$ can be determined based on the sale price of a unit, labor costs, material costs and penalty per shortage unit in customer contracts in real operation context. The total expected system profit in the next mission is defined as the production gain $C_{\mathrm{PG}}$, minus the production loss $C_{\mathrm{PL}}$ and is shown by

$$
\begin{aligned}
& \text { Production Profit } \\
& =\text { Production gain }- \text { Production loss } \\
& =C_{\mathrm{PG}}-C_{\mathrm{PL}} \\
& C_{\mathrm{PG}}=c_{g} \sum_{g_{k} \geq D} g_{k} \frac{1}{\tau} \int_{0}^{\tau} P_{k}(t) d t \\
& C_{\mathrm{PL}}=c_{l} \sum_{g_{k}<D}\left(D-g_{k}\right) \frac{1}{\tau} \int_{0}^{\tau} P_{k}(t) d t
\end{aligned}
$$

where $P_{k}(t)$ is the state probability of the system at time $t$ in the next mission, $\tau$ is the next mission duration and $D$ is the demand level of the system in the next mission.

In (26), the production gain $C_{\mathrm{PG}}$ is calculated for each unit of performance rate that satisfies demand $\left(g_{k}, g_{k} \geq D\right)$. It is a product of the average production gain per unit of performance rate $c_{g}$, the output performance rate of the system at state $k, g_{k}, g_{k} \geq D$, and the expected probability that the system is in state $k$ in the next mission $(1) /(\tau) \int_{0}^{\tau} P_{k}(t) d t$. The production loss $C_{\mathrm{PL}}$ is calculated in a similar way but it is only counted per unit of unsupplied demand $\left(D-g_{k}, g_{k}<D\right)$.

2) Maintenance Cost and Time in the Maintenance Break: For each component $i$, an amount of $c_{i}\left(Y_{i}, X_{i}\right)$ is required to repair it from state $Y_{i}$ to state $X_{i}$ in the maintenance break. If no maintenance action is performed on component $i$, i.e., $X_{i}=Y_{i}, c_{i}\left(Y_{i}, Y_{i}\right)=0$. When $Y_{i}<X_{i}<K$, the maintenance cost of maintenance actions on component $i: c_{i}\left(Y_{i}, X_{i}\right)<$ $c_{i}\left(Y_{i}, K\right)$. The maintenance cost of a component $i$ can be arranged in matrix form as in [15]

$C_{i}=\left[\begin{array}{cccc}0 & c_{i}(0,1) & \cdots & c_{i}(0, K) \\ 0 & 0 & \cdots & c_{i}(1, K) \\ \cdots & \cdots & \cdots & \cdots \\ 0 & 0 & \cdots & c_{i}(K-1, K) \\ 0 & 0 & \ldots & 0\end{array}\right], \quad i=1,2, \ldots, N$

The total system maintenance cost is the summation of maintenance costs of all components

$$
C_{M}(X)=\sum_{i=1}^{N} c_{i}\left(Y_{i}, X_{i}\right)
$$

Similarly, we define $t_{i}\left(Y_{i}, X_{i}\right)$ as the time of repairing component $i$ from state $Y_{i}$ to state $X_{i}$ as

$$
T_{i}=\left[\begin{array}{cccc}
0 & t_{i}(0,1) & \cdots & t_{i}(0, K) \\
0 & 0 & \cdots & t_{i}(1, K) \\
\cdots & \cdots & \cdots & \cdots \\
0 & 0 & \cdots & t_{i}(K-1, K) \\
0 & 0 & \cdots & 0
\end{array}\right], \quad i=1,2, \ldots, N
$$


When the components in the system are repaired one by one, the total system maintenance time can be obtained by taking the sum of all components' repair times as

$$
T(X)=\sum_{i=1}^{N} t_{i}\left(Y_{i}, X_{i}\right) .
$$

In summary, the total system profit (cost) function is calculated from the start of the maintenance break to the end of the next mission as presented in

$$
C=C_{\mathrm{PG}}-C_{\mathrm{PL}}-C_{M}
$$

\section{Selective Maintenance Modeling and Solution Methodology}

The objective of the selective maintenance problem is to find which components to be maintained and how to maintain them during the maintenance break given their conditions at the time of entering the maintenance depot. Thus, a solution of the selective maintenance is a combination of the states of all components at the time of exiting the maintenance depot. It is in vector form $X=\left\{X_{1}, X_{2}, \ldots, X_{N}\right\}$.

The selective maintenance problem can be formulated as a non-linear integer programming problem as follows:

$$
\begin{aligned}
\text { Maximize } & C \\
\text { Subject to : } & R_{s}(X, \tau, D) \geq R_{0} \\
& C_{M}(X) \leq C_{0} \\
& T(X) \leq T_{0} \\
& \text { if } X_{j}>0 \text { then } X_{i}>0 \forall j \in S_{i}^{\mathrm{IF}} \\
& X_{i} \geq X_{i+1} \forall i=1,2, \ldots, N-1 \\
& Y_{i} \leq X_{i} \leq K, X_{i} \text { is integer, } \\
& \forall i=1,2, \ldots, N .
\end{aligned}
$$

In this paper, the maintenance manager has to determine maintenance activities associated with each component to achieve the maintenance objective of maximizing the total profit of the system during the whole next mission under limitation of time and budget for maintenance as well as maintaining the requirement of the system reliability in the next operating mission. The objective function is to maximize the total multistate system profit, which is equal to the production gain in the next mission minus the production loss and minus the maintenance cost. Each element in the cost function, $C$, is calculated as in Section IV-B.

Constraint (35) indicates that the required system reliability in the next operating mission at demand $D$ must be greater than or equal to a specified value $R_{0} . R_{s}(X, \tau, D)$ is calculated using the approach discussed in Section III. Constraints (36) and (37) are two resource constraints, which indicate that the resources for maintenance must be within the available budget and time allotted within the break between the last mission and the upcoming mission. Constraint (38) describes type-1 S-dependence in the system: an affected component $j$ belongs to the set of components affected by component $i, S_{i}^{\mathrm{IF}}$, then component $i$ cannot be in failure state if the state of component $j>0$. Constraint (39) describes type-2 S-dependence in the system: the

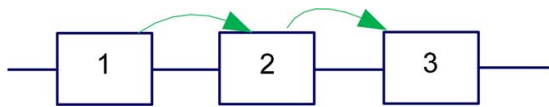

Fig. 5. MSS in example 1.

TABLE I

COMPONENTS' TRANSITION RATES

\begin{tabular}{|c|c|c|c|}
\hline \multirow{2}{*}{$\begin{array}{c}\text { Component } \\
\text { (i) }\end{array}$} & \multicolumn{3}{|c|}{ Transition rate $\lambda_{i, k}$} \\
\cline { 2 - 4 } & $k=3$ & $k=2$ & $k=1$ \\
\hline 1 & 0.12 & 0.18 & 0.15 \\
\hline 2 & 0.09 & 0.15 & 0.05 \\
\hline 3 & 0.17 & 0.12 & 0.2 \\
\hline
\end{tabular}

state of a component is less than or equal to the states of its predecessors. The decision variable, $X$, is a combination of components' states at the beginning of the next mission. Since the maintenance activities do not worsen the state of the components, $X_{i}$ must be an integer value between $Y_{i}$ and the maximum state $K$ for all $i=1,2, \ldots, N$.

In this paper, we use genetic algorithm (GA) to deal with the non-linear selective maintenance optimization model. It is recognized that GA has simple structure and is easy to implement using computer programming. GA has been widely used for various types of optimization problems including maintenance optimization [9], [15], [33].

In GA, we use a chromosome consisting of $N$ genes to represent a solution of the selective maintenance problem. Each element of the state vector corresponds to each gene in GA solution representation. $X_{i}$ is the targeted state of the corresponding component $i$ in the maintenance period. If the states of components at the time of entering the maintenance depot, $Y_{i}$, are known, the MSS profits, maintenance cost and time as well as the system reliability in the next operating mission can be calculated for each vector $X$. Thus, GA can be used to find the best combination of the components' outcome states after the maintenance break for the proposed optimal selective maintenance problem.

\section{EXAMPLES, Results AND DisCUSSIONS}

Example 1: This example is devoted for illustrating the reliability analysis of MSS with type-2 S-dependent components. Consider a MSS consisting of three multistate components connected in series with type-2 S-dependence as presented in Fig. 5. Each component can be in one of four possible states, i.e., $S$ $=\{0,1,2,3\}$ with corresponding performance rates in a set $G=\{0,1,2,3\}$. The nominal state transition rates are provided in Table I and dependent exponents $m_{2}=m_{3}=1$.

All components are as good as new at time $t=0$, find the probability that the system can deliver at least at demand $D=2$ in a mission time $t=1$ year.

Denote the system's state by a set of $\left(s_{1}, s_{2}, s_{3}\right)$ with $s_{i}$ being the state of component $i, i=1,2,3$. The state diagram is constructed as in Fig. 6.

In Fig. 6, state " $F$ " or "Failed" indicates that at least one component in the system is in state 0 , i.e., complete failure state. Once a component is in state 0 , no other components can 


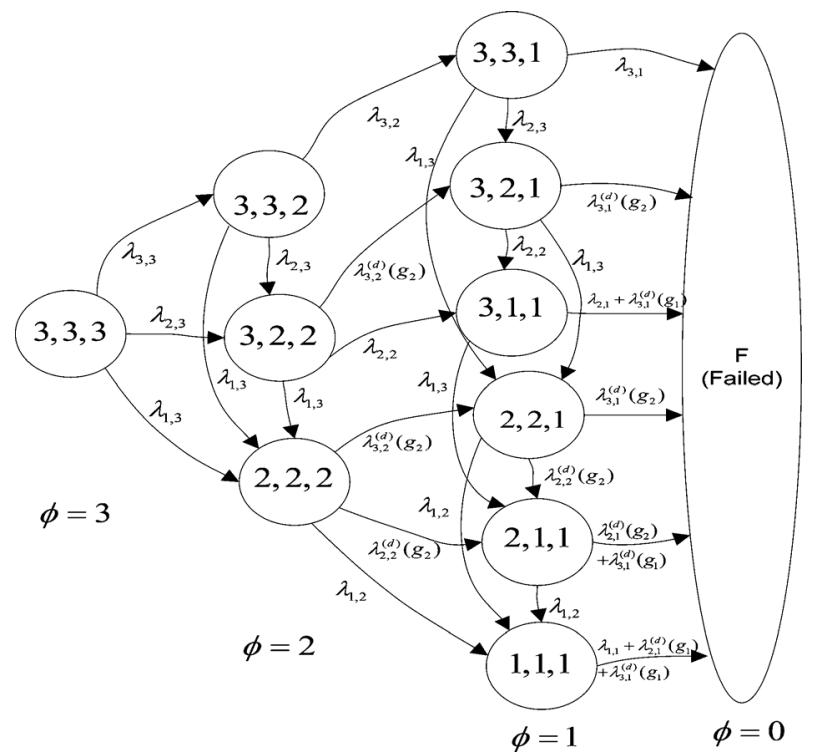

Fig. 6. Transition diagram of a three-component system with type-2 S-dependence.

degrade further in the mission. The C-K system of equations can be written as given by (41), shown at the bottom of the page. In example 1 , the size of matrix $\Lambda$ is $11 \times 11$. The $\mathrm{C}-\mathrm{K}$ system of equations can be solved with initial condition vector $\mathbf{P}(0)=[1,0,0,0,0,0,0,0,0,0,0]$ using the method presented in Section III-C. With $t=1$, we obtain

$$
\begin{aligned}
& \mathbf{P}=\left[\begin{array}{lll}
0.6839 & 0.11920 .06780 .09400 .0070
\end{array}\right. \\
& \left.\begin{array}{lllllll}
0.0029 & 0.0053 & 0.0039 & 0.0050 & 0.0090 & 0.0020
\end{array}\right] \\
& \Rightarrow P_{3}=P_{3,3,3}=0.6839 \\
& P_{2}=P_{3,3,2}+P_{3,2,2}+P_{2,2,2} \\
& =0.1192+0.0678+0.0940=0.2810 \\
& P_{1}=0.0070+0.0029+0.0053+0.0039 \\
& +0.0050+0.0090=0.0331 \\
& P_{0}=0.0020 \text {. }
\end{aligned}
$$

Hence, the system reliability at $D=2$ is

$\Rightarrow R_{s}(D)=\sum_{k=0}^{K} P_{k} \delta\left(g_{k}, D\right)=(0.6839+0.2810)=0.9649$.

Example 2: Consider a MSS consisting of five multistate components in series with their dependence as presented in Fig. 7. Each component can be in one of four possible states $S=\{0,1,2,3\}$ with corresponding performance rates in a

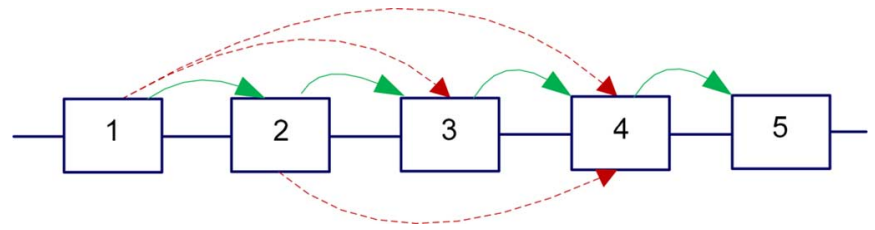

Fig. 7. MSS in Example 2.

TABLE II

COMPONENTS' CHARACTERISTICS IN EXAMPLE 2

\begin{tabular}{|c|c|c|c|c|}
\hline \multirow{2}{*}{$\begin{array}{c}\text { Component } \\
\text { (i) }\end{array}$} & \multicolumn{2}{|c|}{ Transition rate $\lambda_{i, k}$ (year $\left.^{-1}\right)$} & \multirow{2}{*}{$m_{i}$} \\
\cline { 2 - 4 } & $k=3$ & $k=2$ & $k=1$ & \\
\hline 1 & 0.08 & 0.15 & 0.1 & - \\
\hline 2 & 0.06 & 0.11 & 0.05 & 1 \\
\hline 3 & 0.14 & 0.09 & 0.2 & 0.5 \\
\hline 4 & 0.18 & 0.1 & 0.15 & 0.5 \\
\hline 5 & 0.11 & 0.08 & 0.16 & 1 \\
\hline
\end{tabular}

set $G=\{0,1,2,3\}$. The nominal state transition rates and dependence exponents are provided in Table II.

Components 1 and 2 may fail due to induced failure processes with $\lambda_{1}^{\mathrm{IF}}(t)=0.05$ and $\lambda_{2}^{\mathrm{IF}}(t)=0.1 t$. In the set $S^{\mathrm{IF}}=\{1,2\}$, component 1 can cause components 3 and 4 to fail immediately, and component 2 can cause component 4 to fail immediately, i.e., $S_{1}^{\mathrm{IF}}=\{3,4\}, S_{2}^{\mathrm{IF}}=\{4\}$.

Upon arriving at the maintenance depot, the vector of components' states is $Y=[2,0,1,1,0]$.

The repair time (in days) and cost (in $\$ 1000$ ) matrices of each component are given as follows:

$$
\begin{array}{rlr}
C_{1} & =\left[\begin{array}{cccc}
0 & 4.5 & 9 & 14.5 \\
0 & 0 & 4 & 12 \\
0 & 0 & 0 & 9.5 \\
0 & 0 & 0 & 0
\end{array}\right] C_{2}=\left[\begin{array}{cccc}
0 & 3 & 8.5 & 16 \\
0 & 0 & 6 & 12 \\
0 & 0 & 0 & 4.5 \\
0 & 0 & 0 & 0
\end{array}\right] \\
C_{3} & =\left[\begin{array}{cccc}
0 & 2.5 & 6 & 10 \\
0 & 0 & 4 & 8 \\
0 & 0 & 0 & 3.5 \\
0 & 0 & 0 & 0
\end{array}\right] C_{4}=\left[\begin{array}{cccc}
0 & 2.5 & 4.5 & 9 \\
0 & 0 & 3 & 6.8 \\
0 & 0 & 0 & 4 \\
0 & 0 & 0 & 0
\end{array}\right] \\
C_{5} & =\left[\begin{array}{cccc}
0 & 3.5 & 6 & 10 \\
0 & 0 & 3 & 5.5 \\
0 & 0 & 0 & 2 \\
0 & 0 & 0 & 0
\end{array}\right] \\
T_{1} & =\left[\begin{array}{cccc}
0 & 1 & 2.5 & 3.5 \\
0 & 0 & 1.5 & 2 \\
0 & 0 & 0 & 1.5 \\
0 & 0 & 0 & 0
\end{array}\right] & T_{2}=\left[\begin{array}{cccc}
0 & 1.5 & 2 & 5 \\
0 & 0 & 1.5 & 3 \\
0 & 0 & 0 & 1 \\
0 & 0 & 0 & 0
\end{array}\right]
\end{array}
$$

$$
\left\{\begin{array}{l}
\frac{d P_{3,3,3}(t)}{d t}=-\left(\lambda_{1,3}+\lambda_{2,3}+\lambda_{3,3}\right) P_{3,3,3}(t) \\
\frac{d P_{3,3,2}(t)}{d t}=\lambda_{3,3} P_{3,3,3}(t)-\left(\lambda_{1,3}+\lambda_{2,3}+\lambda_{3,2}\right) P_{3,3,2}(t) \\
\frac{d P_{3,2,2}(t)}{d t}=\lambda_{2,3} P_{3,3,3}(t)+\lambda_{2,3} P_{3,3,2}(t)-\left(\lambda_{1,3}+\lambda_{2,2}+\lambda_{3,2}^{(d)}\left(g_{2}\right)\right) P_{3,2,2}(t) \\
\cdots \frac{d P_{F}(t)}{d t}=\lambda_{3,1} P_{3,3,1}(t)+\lambda_{3,1}^{(d)}\left(g_{2}\right) P_{3,2,1}(t)+\cdots+\left(\lambda_{1,1}+\lambda_{2,1}^{(d)}\left(g_{1}\right)+\lambda_{3,2}^{(d)}\left(g_{1}\right)\right) P_{1,1,1}(t)
\end{array}\right.
$$


TABLE IV

SElective MaInTENANCE Results

\begin{tabular}{|c|c|c|c|c|c|c|c|c|c|}
\hline $\begin{array}{c}\text { Maintenance scenario for } \\
\text { the system with }\end{array}$ & $X_{1}$ & $X_{2}$ & $X_{3}$ & $X_{4}$ & $X_{5}$ & $\begin{array}{c}C_{M}(X) \\
(\$ 1,000)\end{array}$ & $\begin{array}{c}T(X) \\
(\text { days })\end{array}$ & $R_{s}(D)$ & $\begin{array}{c}C(X) \\
(\$ 1000)\end{array}$ \\
\hline S-dependent components & 3 & 3 & 2 & 2 & 2 & 38.5 & 12 & 0.85845 & 289.1 \\
\hline Independent components & 3 & 2 & 3 & 3 & 2 & 39 & 12 & 0.90474 & 317.8 \\
\hline
\end{tabular}

TABLE III

SYSTEM INFORMATION AND RESOURCE AVAILABILITIES

\begin{tabular}{|c|c|c|c|c|c|c|}
\hline $\begin{array}{c}c_{g} \\
(\$ 10,000)\end{array}$ & $\begin{array}{c}c_{l} \\
(\$ 10,000)\end{array}$ & $R_{0}$ & $D$ & $\begin{array}{c}\tau \\
\text { (years })\end{array}$ & $\begin{array}{c}C_{0} \\
(\$ 1,000)\end{array}$ & $\begin{array}{c}T_{0} \\
(\text { days })\end{array}$ \\
\hline 20 & 50 & 0.85 & 2 & 0.5 & 40 & 12 \\
\hline
\end{tabular}

$$
\begin{aligned}
T_{3} & =\left[\begin{array}{cccc}
0 & 1 & 2.5 & 5 \\
0 & 0 & 2 & 3.5 \\
0 & 0 & 0 & 1 \\
0 & 0 & 0 & 0
\end{array}\right] T_{4}=\left[\begin{array}{cccc}
0 & 1 & 3 & 4.5 \\
0 & 0 & 1.5 & 3 \\
0 & 0 & 0 & 1.5 \\
0 & 0 & 0 & 0
\end{array}\right] \\
T_{5} & =\left[\begin{array}{cccc}
0 & 1.5 & 2 & 3 \\
0 & 0 & 1 & 2.5 \\
0 & 0 & 0 & 1.5 \\
0 & 0 & 0 & 0
\end{array}\right]
\end{aligned}
$$

The maintenance manager has to find selective maintenance actions to be performed on the system under the requirement and resource availabilities as given in Table III.

The Markov analysis for the system is performed by denoting the state of the system by $\left(s_{1}, s_{2}, s_{3}, s_{4}, s_{5}\right)$. We enumerate all states of the system including $(3,3,3,3,3),(3,3,3,3,2),(3,3$, $3,2,2),(3,3,2,2,2),(3,2,2,2,2),(2,2,2,2,2),(3,3,3,3$, 1), (3, 3, 3, 2, 1), (3, 3, 2, 2, 1), (3, 2, 2, 2, 1), (2, 2, 2, 2, 1), (3, $3,3,1,1),(3,3,2,1,1),(3,3,1,1,1),(3,2,2,1,1),(3,2,1$, $1,1),(3,1,1,1,1),(2,2,2,1,1),(2,2,1,1,1),(2,1,1,1,1)$, $(1,1,1,1,1)$, and state $F$ denoting the complete failure state of the system. The reliability analysis is done in the same way as in Example 1.

Using the given input data, we code and solve the selective maintenance problem for the MSS in MatlabR2012a. The selective maintenance scenarios for the system with S-dependence is compared with the scenario where $\mathrm{S}$-dependence is ignored, as presented in Table IV.

In the first maintenance scenario, the system is subjected to both types of S-dependence. The best maintenance strategy is to repair component 1 and 2 to their perfect functioning state and three other components to state 2 . The expected profit for this maintenance strategy in the next mission considering the average production gain, loss and the cost of maintenance is $\$ 289.1 \mathrm{~K}$.

We also investigate the effect of dependence in the system by solving the selective maintenance problem for the same system and assuming that the components degrade independently. The system reliability and cost elements can be evaluated using the same method but the induced failure rate and dependence exponents are all set to zero; constraints (38) and (39) are removed from the optimization model. The obtained result indicates that the best maintenance strategy is to repair components 1,3 , and 4 to their perfect condition and components 2 and 5 to state 2 . It can be seen that the resources used in the maintenance break

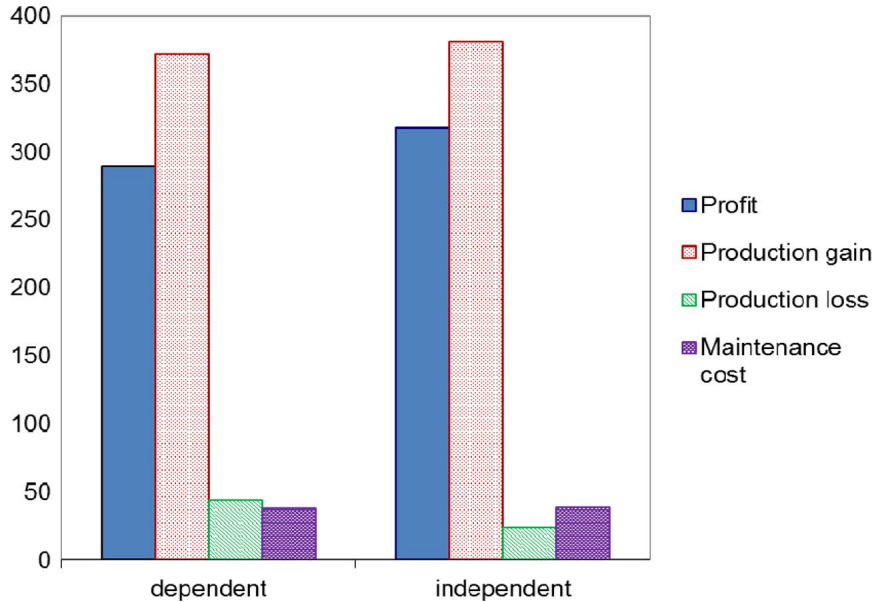

Fig. 8. MSS profit and cost analysis.

for this strategy are not much different from the strategies for dependent systems. However, the system reliability when components are independent is much higher, at 0.90474 , i.e., $5 \%$ larger; and the total profit is also approximately $10 \%$ larger, at $\$ 317.8 \mathrm{~K}$, in comparison with the independent case. Thus, we can conclude that ignoring S-dependence leads to another selective maintenance decision, and this may overestimate the system reliability and profit.

In order to investigate the differences between two maintenance scenarios in Table IV in terms of cost and profit results, we examine the elements in the cost function as in (30) for each optimal strategy. Fig. 8 shows the profit and cost elements of interest.

In the two selective maintenance strategies, the costs for maintenance in the break when components are dependent and when they are independent are not much different. However, when components are dependent, the production loss is much greater $(\sim 20000)$ and the production gain is smaller. Thus, the total system profit is significantly different for the two cases. We can conclude that the two types of S-dependence have negative effects on the reliability and performance of the system. It is reasonable since the system reliability when components are independent is greater than that when components are S-dependent. When the probability of the system being in high states is bigger, it can result in a larger total profit. This result implies that ignoring S-dependence may lead to an optimistic estimation of the system profit.

\section{CONCLUSION}

This paper studies the selective maintenance problem for multistate systems with multistate S-dependent components. 
The dependence relationships between components in series systems are discussed in multistate context. Firstly, the induced failure of a multistate component can immediately cause complete failures of some other components. Secondly, as components in the system deteriorate, the degradation of a component reduces the capacity flowing to the subsequent component, thus affecting the degradation of these components. The repair actions of S-dependent components and the costs in the multistate system are analyzed, and a selective maintenance model is proposed to maximize the total profit of the multistate system subjected to time and reliability and resources constraints.

The example and results imply that S-dependence has significant effect on the MSS reliability. Also, ignoring S-dependence may lead to a wrong determination of selective maintenance plan for the system and overestimation of the system performance. The selective maintenance model can help the maintenance manager make the right decision to maintain the system with available resources and under restricted conditions.

The paper investigates the selective maintenance problem for a multistate series system under a single operating mission. The components in the system degrade gradually. Different system structures and components' degradation and multiple mission selective maintenance for multistate systems with dependent components are suggested for future studies. In addition, the state space of Markov analysis for the system increases exponentially when the number of components and number of states of each component increase. More efficient methods for solving the system model are also recommended for future research.

\section{ACKNOWLEDGMENT}

The authors would like to thank anonymous reviewers for their very constructive comments.

\section{REFERENCES}

[1] H. Wang, "A survey of maintenance policies of deteriorating systems," Eur. J. Oper. Res., vol. 139, no. 3, pp. 469-489, 2002.

[2] A. Sharma, G. Yadava, and S. Deshmukh, "A literature review and future perspectives on maintenance optimization," J. Quality Maint. Eng., vol. 17, no. 1, pp. 5-25, 2011.

[3] R. P. Nicolai and R. Dekker, "Optimal maintenance policies of multicomponent systems, a review," in Complex System Maintenance Handbook, ser. Series in Rel. Eng.. Berlin, Germany: Springer, 2008, pp. 263-286.

[4] W. F. Rice, C. R. Cassady, and J. A. Nachlas, "Optimal maintenance plans under limited maintenance time," in Proc. Ind. Eng. Res. Conf., 1998, pp. 1-8.

[5] C. R. Cassady, W. P. Murdock, and E. A. Pohl, "Selective maintenance for support equipment involving multiple maintenance actions," J. Oper. Res., vol. 129 , no. 2, pp. 252-258, 2001.

[6] L. M. Maillart, C. R. Cassady, C. Rainwater, and K. Schneider, "Selective maintenance decision-making over extended planning horizons," IEEE Trans. Rel., vol. 58, no. 3, pp. 462-469, Jun. 2009.

[7] H. Zhu, F. Liu, X. Shao, Q. Liu, and Y. Deng, "A cost-based selective maintenance decision-making method for machining line," Quality Rel. Eng. Int., vol. 27, no. 2, pp. 191-201, 2011.

[8] M. Pandey, M. J. Zuo, R. Moghaddas, and M. K. Tiwari, "Selective maintenance for binary systems under imperfect repair," Rel. Eng. Syst. Safety, vol. 113, pp. 42-51, 2013.

[9] Y. Liu and H. Z. Huang, "Optimal selective maintenance strategy for multi-state systems under imperfect maintenance," IEEE Trans. Rel., vol. 59, no. 2, pp. 356-367, Feb. 2010.

[10] G. Maaroufi, A. Chelbi, and N. Rezg, "A selective maintenance policy for multi-component systems with stochastic and economic dependence," in Proc. 9th Int. Conf. Modelling, Optimization and Simulation, 2012.
[11] A. Lisnianski and G. Levitin, Multi-State System Reliability: Assessment, Optimization and Applications. Singapore: World Scientific, 2003.

[12] B. Natvig, Multi-State Systems Reliability Theory With Applications. Hoboken, NJ, USA: Wiley, 2011.

[13] C. Chen, M. Q.-H. Meng, and M. J. Zuo, "Selective maintenance optimization for multi-state systems," in Proc. IEEE Can. Conf. Electr. Comput. Eng., 1999, pp. 1477-1482.

[14] M. Pandey, M. J. Zuo, and R. Moghaddas, "Selective maintenance for multistate system with multistate components," IIE Trans., vol. 45, pp. 1221-1234, 2014.

[15] C. D. Dao, M. J. Zuo, and M. Pandey, "Selective maintenance for multistate series-Parallel systems under economic dependence," Rel. Eng. Syst. Safety, vol. 121, pp. 240-249, 2014.

[16] L. Cui and H. Li, "Analytical method for reliability and MTTF assessment of coherent system with dependent components," Rel. Eng. Syst. Safety, vol. 92, pp. 300-307, 2007.

[17] Y. Sun, L. Ma, J. Mathew, and S. Zhang, "An analytical model for interactive failure," Rel. Eng. Syst. Safety, vol. 91, pp. 495-504, 2006.

[18] D. N. P. Murthy and D. G. Nguyen, "Study of a two-component system with failure interaction," Naval Res. Logistics, vol. 10, pp. 239-247, 1985a.

[19] D. N. P. Murthy and D. G. Nguyen, "Study of a multi-component system with failure interaction," Eur. J. Oper. Res., vol. 21, pp. 330-338, 1985b.

[20] P. A. Scarf and M. Deara, "On the development and application of maintenance policies for a two-component system with failure dependence," IMA J. Manag. Math., vol. 9, no. 2, pp. 91-107, 1998.

[21] P. A. Scarf and M. Deara, "Block replacement policies for a two-component system with failure dependence," Naval Res. Logistics, vol. 50, pp. 70-87, 2003.

[22] A. Barros, A. Bérenguer, and A. Grall, "Optimization of replacement times using imperfect monitoring information," IEEE Trans. Rel., vol. 52, no. 4, pp. 523-533, Dec. 2003.

[23] M. T. Lai, "Periodical replacement model for a multi-unit system subject to failure rate interaction," Quality \& Quantity, vol. 41, pp. 401-411, 2007.

[24] C. K. Sung, S. Sheu, T. S. Hsu, and Y. C. Chen, "Extended optimal replacement policy for a two-unit system with failure rate interaction and external shocks," Int. J. Syst. Sci., vol. 40, no. 5, pp. 877-888, 2013.

[25] S. L. Albin and S. Chao, "Preventive replacement in systems with dependent components," IEEE Trans. Rel., vol. 41, no. 2, pp. 230-238, Jun. 1995

[26] N. Rasmekonen and A. K. Parlikad, "Maintenance optimization for assets systems with dependent degradation," IEEE Trans. Rel., vol. 62, no. 2, pp. 362-367, Jun. 2013.

[27] G. Levitin and L. Xing, "Reliability and performance of multistate systems with propagated failures having selective effect," Rel. Eng. Syst. Safety, vol. 95, pp. 655-661, 2010.

[28] G. Levitin, L. Xing, H. Ben-Haim, and Y. Dai, "Multi-state systems with selective propagated failures and imperfect individual and group protections," Rel. Eng. Syst. Safety, vol. 96, no. 12, pp. 1657-1666, 2011.

[29] G. Levitin, "A universal generating function approach for the analysis of multi-state systems with dependent elements," Rel. Eng. Syst. Safety, vol. 84, pp. 285-292, 2004.

[30] C. D. Dao and M. J. Zuo, "Reliability analysis of multi-state systems with S-dependent components," in Proc. RAMS, 2015, pp. 24-29.

[31] S.-H. Sheu and Z. G. Zhang, "An optimal age replacement policy for multi-state systems," IEEE Trans. Rel., vol. 62, no. 1, pp. 1-14, Mar. 2013.

[32] M. Amiri, F. Ghassemi-Tari, A. Mohtashami, and J. S. Sadaghiani, “A methodology for analyzing the transient availability and survivability of a system with the standby components in two cases: The identical components and the non-identical components," J. Appl. Sci., vol. 8, no. 22, pp. 4105-4112, 2008.

[33] S. N. Sivanandam and S. N. Deepa, Introduction to Genetic Algorithms. New York, NY, USA: Springer, 2010.

Cuong D. Dao (S'14) received the B.Sc. degree in mechanical engineering from Hanoi University of Technology, Hanoi, Vietnam, in 2006, and the M.Sc. degree in industrial and manufacturing engineering from Asian Institute of Technology, Thailand, in 2009. He is currently working toward the Ph.D. degree in mechanical engineering at the University of Alberta, Edmonton, AB, Canada.

His research interests include multistate system reliability analysis and selective maintenance modeling. 
Ming J. Zuo (SM'00) received the B.S. degree in agricultural engineering from Shandong Institute of Technology, Shandong, China, in 1982, and the M.S. and $\mathrm{Ph} . \mathrm{D}$. degrees in industrial engineering from Iowa State University, Ames, IA, USA, in 1986 and 1989, respectively.

He is currently a Full Professor with the Department of Mechanical Engineering, the University of Alberta, Edmonton, AB, Canada. His research interests include system reliability analysis, maintenance modeling and optimization, signal processing, and fault diagnosis.

Dr. Zuo is a Fellow of the Institute of Industrial Engineers (IIE) and the Engineering Institute of Canada (EIC) and a Founding Fellow of the International Society of Engineering Asset Management (ISEAM). He is an associate editor for the IEEE TRANSACTIONS ON RELIABILITY, department editor of IIE Transactions (2005-2008, 2011-present), regional editor for North and South American regions for International Journal of Strategic Engineering Asset Management, and a member of the editorial board Reliability Engineering and System Safety, Journal of Traffic and Transportation Engineering, International Journal of Quality, Reliability and Safety Engineering, and International Journal of Performability Engineering. 